\title{
LED mixed lighting device of color temperature and brightness wireless control
}

\author{
Xiaojin YAN ${ }^{1, a}$, Qian LIU ${ }^{1, b}$, Shuying ZHANG ${ }^{1, \mathrm{c}}$ and Dan PENG ${ }^{1, \mathrm{~d}}$ \\ ${ }^{1}$ School of Electrical and Control Engineering, North China Institute of Aerospace Engineering, \\ Langfang, 065000,China \\ a yanxiaojin1018@163.com, ${ }^{\mathrm{b}} 654082702 @ q q . c o m,{ }^{\mathrm{c}}$ 18731601904@163.com, \\ d pengd0092@126.com
}

\begin{abstract}
Keywords: color temperature, brightness, ZigBee, PWM, LED.
Abstract. In view of the white LED problems being medium and high color temperature, the poor CRI of warm color temperature, and single control on the market, a plan is given based on ZigBee wireless communication technology of the LED color temperature and brightness independent control system. Through the wireless sensor network, controls the MCU to produce PWM signal, controls the duty cycle of each LED current, and makes each color of LED mixing to a desired color temperature and brightness. The device not only realizes the LED color temperature and brightness adjustable independently, but also has the advantages of high photosynthetic efficiency and high color rendering.
\end{abstract}

\section{Introduction}

Color temperature is an important Performance index of the LED light source, and affects people's psychological and physiological. At present, most adjustable color temperature of white LED lighting is based on the principle of three primary colors by configuring the red, blue, green three colors light source. At the same time of this way adjusting the color temperature, the brightness of the light source is not constant, and changes as well [1,2]. As a dynamic lighting source, the LED can realize the collocation of color and light, and energy conservation and environmental protection through the intelligent control. As short distance wireless communication technology, the ZigBee technology is convenient for networking, and can access 254 nodes at the same time [3]. In addition, it also has low power consumption, low operation cost, short time delay and high safety performance, etc, and is very suitable for application in industrial control occasions.

This article is based on a study of the effects on people generated from the LED mixed lighting color temperature and brightness changes, analyzes the current LED mixed lighting and control technical defects and deficiencies in detail, designs a device based on ZigBee wireless technology of the LED color temperature and brightness adjustable independently, and the indoor LED mixed lighting device has the high photosynthetic efficiency and high color rendering index (CRI).

\section{System scheme and principle}

The LED mixed lighting light source uses the amber white, red, and blue three colors for the different proportions of mixed to produce white light, and used the single chip microcomputer to control the LED current independently, by changing the proportion between different colors, mixing it into specified color temperature value. Under the color temperature constant, the light is changed by adjusting the proportion of each color. The system uses the wireless control technology to realize intelligent and wiring arbitrarily and solve the limitations of traditional wired lighting system. The main system includes main control unit and auxiliary control unit, the main control unit mainly includes ZigBee wireless sending module, main control MCU, key addressing circuit, display circuit, etc; the auxiliary control unit is made up of the ZigBee wireless receiving module, a single MCU and LED constant current drive circuit, etc. The overall structure is given in Fig.1.

The main control unit completes the collection of parameters such as indoor brightness and keyboard commands, after processing into MCU, broadcast out by the ZigBee sending module, after 
the ZigBee receiving module received the information, it will be sent to the single MCU to control the On, off, and the separate control of the color temperature and brightness of LED lights.

In addition, the main control unit will show each state of the LED lamps and lanterns, color temperature, brightness and other information.

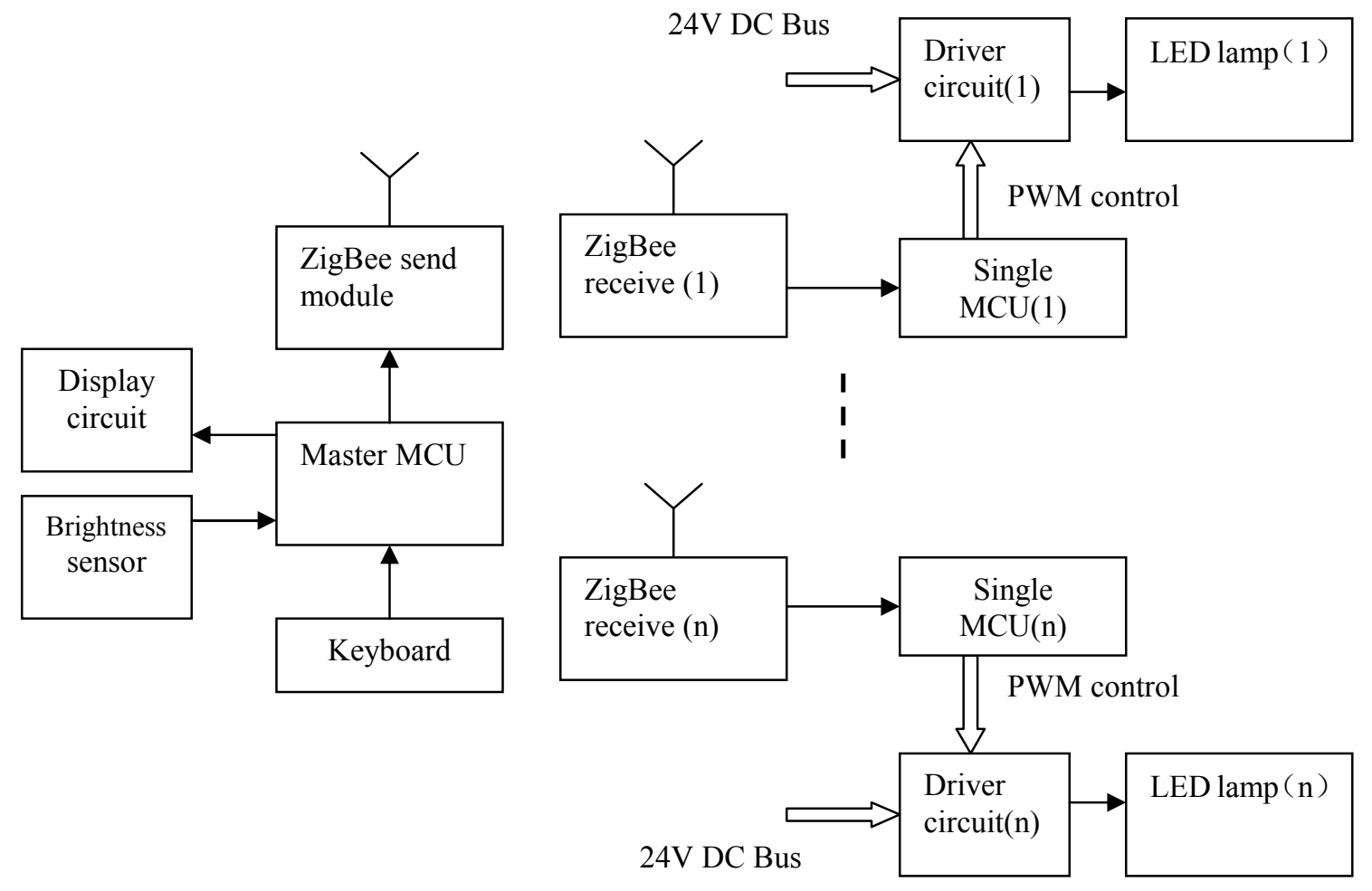

Fig.1 System overall structure diagram

\section{System hardware circuit design}

The control circuit. The CPU of the control circuit uses the single chip microcomputer of STC89C54, which has the characteristics of high cost-effective, low power consumption, LCD display and keys easy control, etc. Through the single chip microcomputer LED brightness and color temperature parameters are set, and then the information is transmitted to the wireless sending module. The minimum system circuit is shown in Fig.2.

The auxiliary CPU control circuit also uses the single chip microcomputer of STC89C54 as the core, similar to the circuit principle diagram of Fig.2, and exports the three independent PWM controls LED of color temperature and brightness.

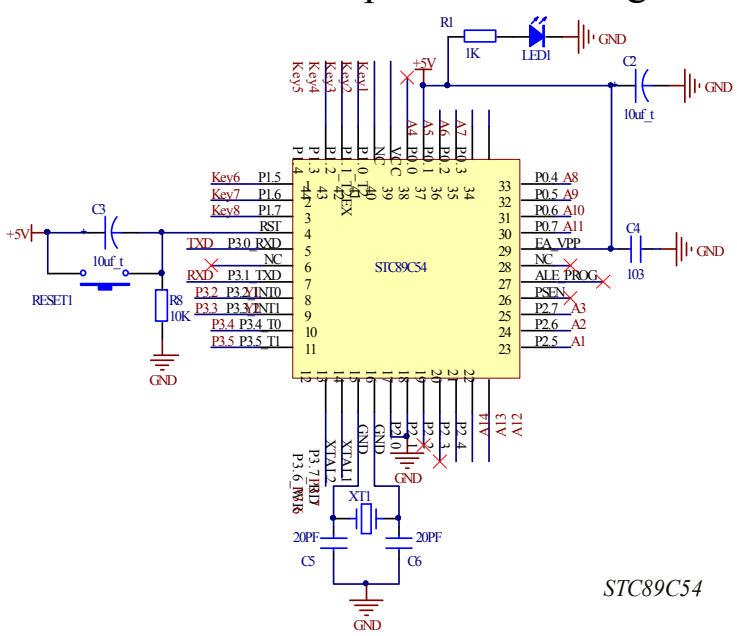

Fig.2 The minimum system of STC89C54

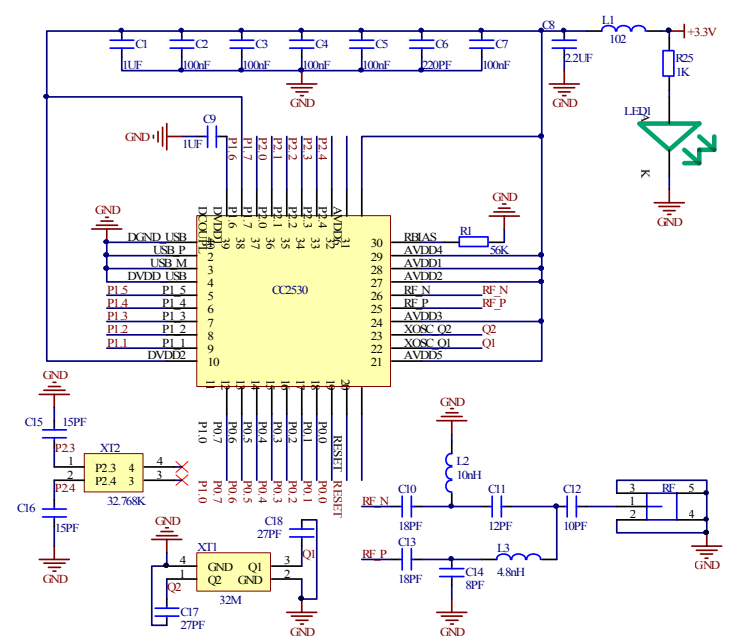

Fig.3 Wireless sending and receiving modules of CC2530 
The wireless receiving and sending circuit. The chip CC2530 is the core of ZigBee wireless sending and receiving module. The chip is not only an enhanced MCU, but also has the special function of wireless transmission. In the process of wireless transmission, it can be transmitted in the form of internet of things, achieve network in hundreds of meters distance, and has the through-wall function, which not only receives the master CPU control instruction, but also can receive the transmitting module receiving real-time information. The circuit is shown in Fig.3.

LED driver circuit. Indoor LED lighting using 24V DC bus for power supply. LED lamp is composed of three different colors of the LED, In order to make a variety of colors of the LED in the best condition, its driving circuit using three-way drive circuit for the LED of each color, according to the number and the voltage of each color LED, the drive topology circuit using Buck and Boost circuit. Because of the ratio of three colors, amber white, red and blue, its output contains one Boost circuit and two Buck circuits. Boost circuit output voltage is 45 Vand output current is $350 \mathrm{~mA}$; one of the buck circuits output voltage is $17.6 \mathrm{~V}$ and another is output $12 \mathrm{~V}$, the output current are both $350 \mathrm{~mA}$. The specific design can refer to reference [4].

\section{System software design}

Master program is mainly used to adjust the white amber, red and blue three colors of LED brightness and color temperature, and display brightness and color temperature values through the LCD screen, and then send the data from the ZigBee module to the auxiliary control panel's receiver module, thus achieving the purposes of real-time dimming and color temperature adjustment. The main control program flow chart is shown in Fig.4.

Auxiliary control program is mainly to receive the data sent from the master, and then the data processed by the CPU, eventually produce the corresponding LED current controlled by the PWM, then achieving the purposes of brightness and color temperature. Auxiliary control program flow chart is shown in Fig.5.

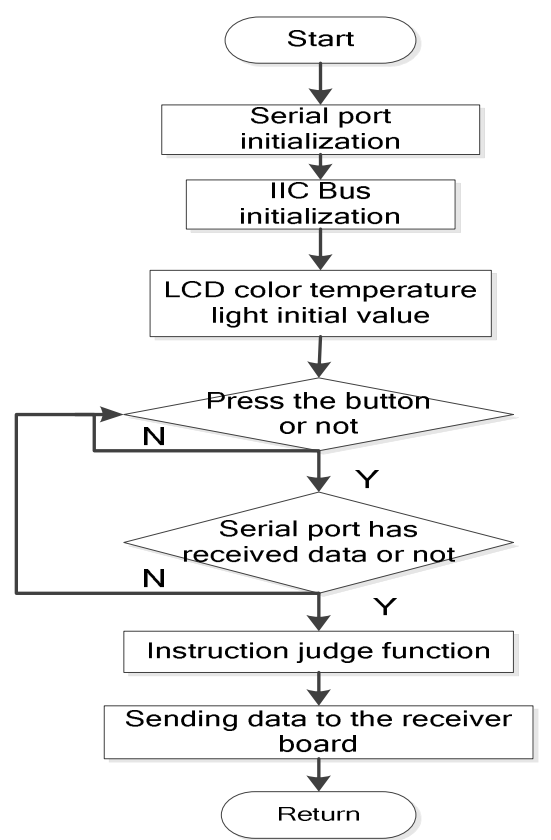

Fig.4 The flow chart of main control program

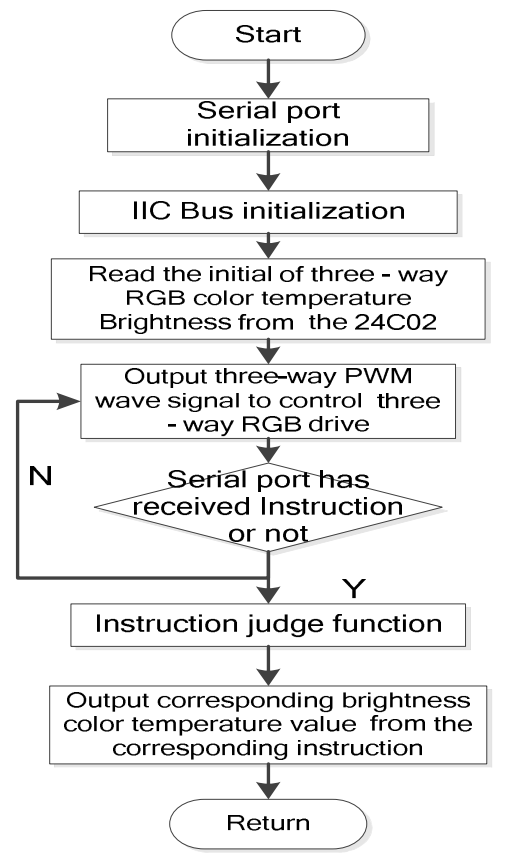

Fig. 5 The flow chart of auxiliary control program

\section{Test results and analysis}

Test results of $4400 \mathrm{~K}, 4200 \mathrm{~K}, 3700 \mathrm{~K}$ color temperature are shown in Fig.6, Fig.7 and Fig.8. The actual color temperature test value at the "+" cursor position, and the theoretical value should be on the curve near "+" cursor, we can see the actual test value is very close to the theoretical value, to achieve the desired effect. The color temperature and brightness change test results is shown in Fig.9. 
In addition, the CRI of test results is higher than 90, and can reflect the change in color temperature and luminance.

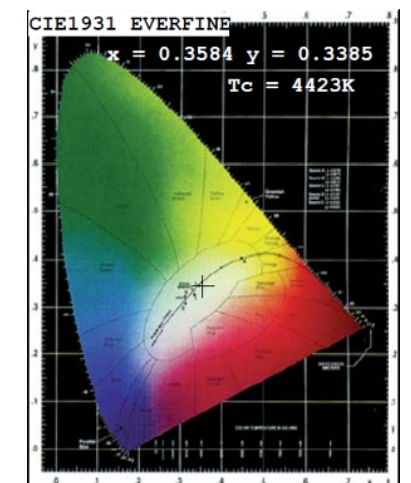

Fig.6 4400K test pattern

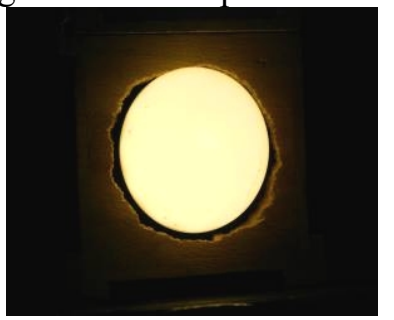

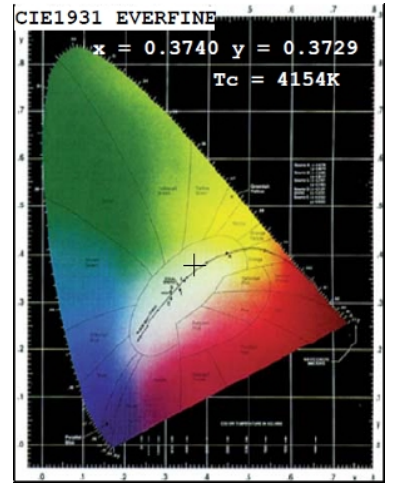

Fig.7 $4200 \mathrm{~K}$ test pattern

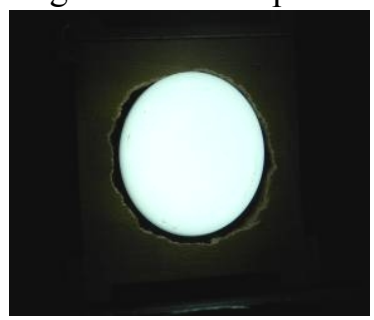

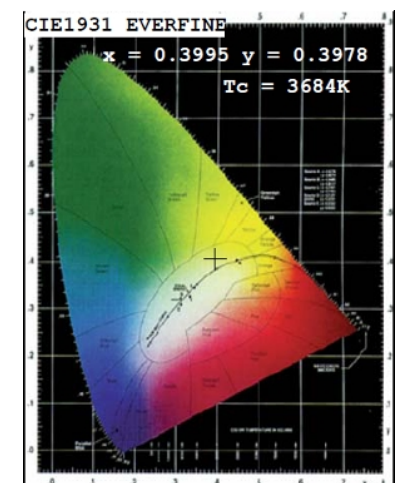

Fig.8 3700K test pattern

Fig.9 Color temperature and brightness change test pattern

\section{Conclusions}

The design combine LED lighting technology and ZigBee wireless intelligent control technology as a whole, make the most advantage of two technologies as possible, and has met the new requirements of LED interior lighting for color temperature, brightness, CRI and other indicators. The device can be used in home interior lighting, shopping malls, conference halls and other occasions, it also easy to form a network, and has broad application prospects.

\section{Acknowledgements}

This work was financially supported by the 2012 Hebei Province Department of Education Science and Technology Research Project (No.: Q2012133), Langfang City Science and Technology Support project (No.:2014011053) and North China Institute of Aerospace Engineering youth fund project (No.: KY-2014-01). At the same time, thanks to the support of Hebei provincial key disciplines "detection technology and automation" and Hebei province equipment manufacturing digitalization and production process automation application technology development center.

\section{References}

[1] He Xin, Cao Guanying, Zou Nianyu, et al: J. 2011 Green Lighting Scientific Development Technology Symposium and the fourth trilateral meeting. (2011), p.188 (In Chinese)

[2] Chun-Wen Tang, Bin-Juine Huang: J. IEEE TRANSA CTIONSON POWER ELECTRONICS.Vol. 25 (2010), p.417

[3] David Egan: J. IEE ComPuting\&Control Engineering. (2005), p.14

[4] Yan Xiaojin: J. Journal of North China Institute of Aerospace Engineering. Vol.23 (2013), p.23 (In Chinese) 